\title{
Mitochondrial DNA leakage induces odontoblast inflammation via the cGAS-STING pathway
}

Lu Zhou' ${ }^{1}$, Yi-Fei Zhang ${ }^{1}$, Fu-Hua Yang ${ }^{1}$, Han-Qing Mao ${ }^{1}$, Zhi Chen ${ }^{1,2}$ and Lu Zhang ${ }^{1,2^{*}}$ (i)

\begin{abstract}
Background: Mitochondrial DNA (mtDNA) is a vital driver of inflammation when it leaks from damaged mitochondria into the cytosol. mtDNA stress may contribute to cyclic GMP-AMP synthase (cGAS) stimulator of interferon genes (STING) pathway activation in infectious diseases. Odontoblasts are the first cells challenged by cariogenic bacteria and involved in maintenance of the pulp immune and inflammatory responses to dentine-invading pathogens. In this study, we investigated that mtDNA as an important inflammatory driver participated in defending against bacterial invasion via CGAS-STING pathway in odontoblasts.

Methods: The normal tissues, caries tissues and pulpitis tissues were measured by western blotting and immunohistochemical staining. Pulpitis model was built in vitro to evaluated the effect of the CGAS-STING pathway in odontoblast-like cell line (mDPC6T) under inflammation. Western blot and real-time PCR were performed to detect the expression of CGAS-STING pathway and pro-inflammatory cytokines. The mitochondrial function was evaluated reactive oxygen species (ROS) generated by mitochondria using MitoSOX Red dye staining. Cytosolic DNA was assessed by immunofluorescent staining and real-time PCR in mDPC6T cells after LPS stimulation. Furthermore, mDPC6T cells were treated with ethidium bromide (EtBr) to deplete mtDNA or transfected with isolated mtDNA. The expression of cGAS-STING pathway and pro-inflammatory cytokines were measured.
\end{abstract}

Results: The high expression of CGAS and STING in caries and pulpitis tissues in patients, which was associated with inflammatory progression. The cGAS-STING pathway was activated in inflamed mDPC6T. STING knockdown inhibited the nuclear import of p65 and IRF3 and restricted the secretion of the inflammatory cytokines CXCL10 and IL-6 induced by LPS. LPS caused mitochondrial damage in mDPC6T, which promoted mtDNA leakage into the cytosol. Depletion of mtDNA inhibited the cGAS-STING pathway and nuclear translocation of p65 and IRF3. Moreover, repletion of mtDNA rescued the inflammatory response, which was inhibited by STING knockdown.

Conclusion: Our study systematically identified a novel mechanism of LPS-induced odontoblast inflammation, which involved mtDNA leakage from damaged mitochondria into the cytosol stimulating the cGAS-STING pathway and the inflammatory cytokines IL-6 and CXCL10 secretion. The mtDNA-cGAS-STING axis could be a potent therapeutic target to prevent severe bacterial inflammation in pulpitis.

\footnotetext{
*Correspondence: luzhang2012@whu.edu.cn

${ }^{1}$ The State Key Laboratory Breeding Base of Basic Science of Stomatology

(Hubei-MOST) and Key Laboratory of Oral Biomedicine Ministry

of Education, School and Hospital of Stomatology, Wuhan University,

Wuhan, China

Full list of author information is available at the end of the article
} permits use, sharing, adaptation, distribution and reproduction in any medium or format, as long as you give appropriate credit to the original author(s) and the source, provide a link to the Creative Commons licence, and indicate if changes were made. The images or other third party material in this article are included in the article's Creative Commons licence, unless indicated otherwise in a credit line to the material. If material is not included in the article's Creative Commons licence and your intended use is not permitted by statutory regulation or exceeds the permitted use, you will need to obtain permission directly from the copyright holder. To view a copy of this licence, visit http://creativecommons.org/licenses/by/4.0/. The Creative Commons Public Domain Dedication waiver (http://creativeco mmons.org/publicdomain/zero/1.0/) applies to the data made available in this article, unless otherwise stated in a credit line to the data. 
Keywords: Mitochondrial DNA, Mitochondrial damage, cGAS-STING pathway, Odontoblasts, Inflammation, Immune response

\section{Background}

According to Global Burden of Diseases, Injuries, and Risk Factors Study 2016 data, the greatest prevalence was dental caries among all disease and its global incidence was the second [1]. Dental caries is a bacterial infectious disease in hard tissue, which lead to tooth defects and even pulpitis, causing severe pain and seriously affecting the quality of life of patients. Odontoblasts play a crucial role in maintaining a balanced pulp microenvironment to regulate transcellular transport between the pulp and dentine [2]. During the progression of caries, odontoblasts can recognize and respond to bacteria, and these cells are the first line of defense against cariogenic bacteria for their specific localization at the pulp-dentin interface [3]. Thus, it is of importance to elucidate the mechanisms by which odontoblasts eliminate invading bacteria.

The initial function of the cGAS-STING pathway is host defense, but recent studies have revealed its fundamental roles in the development of a variety of inflammatory diseases [4-6]. Cyclic GMP-AMP (cGAMP) synthase (cGAS) can detect intracellular DNA and generate the second messenger cGAMP, thereby activating STING in the endoplasmic reticulum [7]. Then, activated STING is transported from the ER to the Golgi, where it forms a complex. This complex with TANK-binding kinase 1 (TBK1) is then transferred to the endolysosome, in which it stimulates transcription factors such as nuclear factor kappa-B (NF- $\mathrm{kB}$ ) and interferon regulatory factor 3 (IRF3). These transcription factors facilitate the expression of type I interferons or proinflammatory cytokines [8-10]. Emerging evidence shows that the cGAS-STING pathway is activated not only by nonself DNA, such as DNA from viruses, bacteria, and protozoa but also by self-DNA, including intracellular, mitochondrial and nuclear DNA, which can enter the cytoplasm [6].

Mitochondrial DNA (mtDNA), which is the only nonnuclear genome, is double-stranded circular DNA (16.5 $\mathrm{kbp}$ ), is a component of mitochondria [11]. mtDNA encodes 37 genes, all of which are associated with oxidative phosphorylation and normal mitochondrial function [12]. Compared with nuclear DNA (nDNA), mtDNA is more unstable and vulnerable to the effects of oxidative stress due to its close proximity to mitochondrial reactive oxygen species (mtROS) and a lack of repair machinery [13, 14]. Accumulating evidence indicates that when mtDNA leaks from damaged mitochondria into the cytosol, it becomes a vital driver of inflammation $[11,15,16]$. mtDNA serves as a kind of mitochondrial danger-associated molecular patterns (mtDAMPs) that can engage various pattern recognition receptors (PRRs) and activate the innate immune system, including cGASSTING $[15,17,18]$.

mtDNA stress may contribute to cGAS-STING pathway activation and type I IFN responses in various pathological states, including infectious diseases, cancer, neurodegeneration and other mitochondriarelated illnesses $[15,16]$. Systemic lupus erythematosus (SLE) neutrophils release mtDNA, which activates the cGAS-STING pathway to promote type I IFN-regulated autoimmunity [19]. Microorganisms such as Mycobacterium tuberculosis can stimulate cGAS activation for mitochondrial damage and mtDNA release, which enhances intracellular survival [20]. Beyond stimulating $\mathrm{T}$ cell proliferation, activation of the cGAS-STING pathway causes tumor vascular collapse and contributes to tumor cell death and apoptosis, promoting the release of tumor-associated antigens [6]. Despite numerous studies describing the importance of the cGAS-STING pathway in various pathological processes, it is still unclear whether the cGAS-STING pathway is involved in inflamed odontoblasts.

The present study identified a distinct mechanism of LPS-induced odontoblast inflammation, which involved the cGAS-STING pathway. Mitochondrial dysfunction and mtDNA leakage into the cytosol were induced by LPS via the cGAS-STING pathway, leading to odontoblast-like cell inflammation. The mtDNA-cGAS-STING axis is thus a crucial regulator of odontoblast inflammation and could be a potent therapeutic target to prevent severe bacterial inflammation.

\section{Methods}

The Additional file provides primer sequences, antibodies and siRNA sequences.

\section{Human dental pulp samples}

Written informed consent was obtained from the patients at the School and Hospital of Stomatology, Wuhan University, and the study was approved by the Institutional Ethics Board of Wuhan University. In this study, tissue samples were obtained from 18 patients, including 6 healthy patients, 6 carious patients, and 6 pulpitis patients. The healthy teeth group consisted of third molars extracted for orthodontics, without any 
caries lesions or periodontitis. The carious teeth group consisted of teeth collected from patients diagnosed with caries and carious lesions but without any history of spontaneous pain. The pulpitis group consisted of teeth collected from patients diagnosed with irreversible pulpitis with spontaneous pain, heat- or cold-stimulating pain and pain at night $[21,22]$.

\section{Cell culture}

The preodontoblast cell line $\mathrm{mDPC} 6 \mathrm{~T}$ is a cell line that was established by our laboratory [23]. mDPC6T cells were cultured in Dulbecco's modified Eagle medium (DMEM, HyClone) supplemented with $10 \%$ fetal bovine serum (FBS, Gibco) at $37{ }^{\circ} \mathrm{C}$ in a humidified $5 \% \mathrm{CO}_{2}$ atmosphere. LPS (Sigma) was added to the medium to establish an LPS-induced odontoblast injury model. Ethidium bromide (EtBr, Thermo Fisher Scientific) was used to deplete mtDNA and was incubated with the cells for $48 \mathrm{~h}(1 \mu \mathrm{g} / \mathrm{ml})$ as described.

\section{RNA preparation and real-time PCR}

A total RNA miniprep kit (Axygen) was used to extract total RNA from mDPC6T cells according to the manufacturer's instructions. Real-time PCR was performed as previously described. The primer sequences are listed in the Additional file.

\section{Protein extraction and western blotting}

Total protein samples were collected from mDPC6T cells and tissues using RIPA buffer (Beyotime) containing a phosphatase inhibitor cocktail and protease inhibitor cocktail (MCE). Cytosolic and nuclear protein fractions were isolated by a nuclear and cytoplasmic protein extraction kit (Beyotime). Western blotting was performed according to a previous procedure [24]. The information for the antibodies used is listed in the Additional file.

\section{RNA interference}

siRNAs were purchased from Genepharma (China, Shanghai). mDPC6T cells were transfected with $20 \mathrm{nM}$ siRNA and Lipofectamine 3000 (Invitrogen) according to the manufacturer's protocol. Two days later, mDPC6T cells were transfected with siRNAs again and treated with LPS. Nonspecific siRNA was used as a negative control. The siRNA sequences used in this study are shown in the Additional file.

\section{Immunocytochemistry}

All 3 groups of human tooth samples were demineralized in 10\% EDTA buffer for 3 months and then embedded in paraffin. The samples were analyzed according to a previous protocol, and the staining process was performed as previously described [25]. The antibodies used are shown in the Additional file.

\section{DNA isolation and mtDNA copy number analysis}

The cytosol of mDPC6T cells was extracted with the Mitochondria Isolation Kit (Beyotime) according to the manufacturer's protocol. The cytosolic supernatant was collected without mitochondria. The genomic DNA miniprep kit (Axygen) was used to isolate the DNA from the collected cytoplasm supernatant. DNA (10 ng) was subjected to real-time PCR on a CFX96 system (BioRad) with SYBR qPCR master mix (Vazyme). The short regions of the tRNA-Leu ${ }^{\mathrm{UUR}}$ and $\beta 2$-microglobulin genes were amplified to determine mitochondrial DNA and nuclear DNA, respectively, as previously described [26]. The $\mathrm{mtDNA} / \mathrm{nDNA}$ ratio was calculated to determine the mtDNA copy number. All primers are listed in the Additional file.

\section{mtDNA isolation and transfection}

mtDNA was isolated from mDPC6T cells using a mitochondrial DNA isolation kit (BioVision) according to the protocol. The extracted mtDNA was resuspended in TE buffer and stored at $-20^{\circ} \mathrm{C}$ for future use. mDPC6T cells were seeded in 6-well plates and transfected with mtDNA $(1 \mu \mathrm{g} /$ well) and Lipofectamine 3000 (Thermo Fisher Scientific) according to the manufacturer's instructions.

\section{mtROS measurement}

MitoSOX Red dye (Invitrogen; M36008) was used to measure mtROS according to the manufacturer's protocol. mDPC6T cells were stained with $5 \mu \mathrm{M}$ of MitoSOX Red Dye for $10 \mathrm{~min}$ after treated with LPS $(20 \mu \mathrm{g} / \mathrm{ml})$ for $24 \mathrm{~h}$. After staining, cells were washed with PBS and fixed in paraformaldehyde for $10 \mathrm{~min}$. Then, mDPC6T cells were counterstained with DAPI for $10 \mathrm{~min}$. Stained cells were observed and photographed on a confocal microscope (Olympus, Japan).

\section{Immunofluorescence analysis}

mDPC6T cells were seeded in confocal dishes and treated with LPS $(20 \mu \mathrm{g} / \mathrm{ml})$ for $24 \mathrm{~h}$. Next, mDPC6T cells were incubated with MitoTracker (Invitrogen) using a working concentration of $200 \mathrm{nM}$ for $15 \mathrm{~min}$. After staining, mDPC6T cells were fixed in paraformaldehyde and permeabilized with $0.1 \%$ Triton X-100. After $10 \%$ bovine serum albumin blocking, mDPC6T were incubated with primary antibodies overnight at $4{ }^{\circ} \mathrm{C}$. Alexa Fluor 488 secondary antibodies were used for signal detection. Labeled mDPC6T cells were observed with a confocal 
microscope (Olympus, Japan). The antibodies used are shown in the Additional file.

\section{Statistical analysis}

Statistical analysis was performed using Student's t-tests or one-way analysis of variance at a significance level of $\mathrm{p}<0.05$ using GraphPad Prism 8. All data are presented as the means \pm standard deviations and are representative of at least three independent experiments. The quantification of western blot results was performed using Fiji software.

\section{Results}

High expression of CGAS and STING was detected in caries and pulpitis tissues

To investigate the clinical importance of cGAS and STING in caries and pulpitis patients, we compared cGAS and STING expression levels in caries, pulpitis and normal tooth samples by western blotting (Fig. 1a) and immunohistochemistry (Fig. 1b, c). The western blotting results showed that the expression of cGAS and STING in pulpitis tissues was much higher than that in normal tissues and caries tissues (Fig. 1a). We also found high expression levels of cGAS and STING mainly in the odontoblast layer in caries tooth samples and pulpitis tooth samples compared with healthy tooth samples (Fig. 1b, c). Moreover, the expression of cGAS and STING was significantly increased with progressive a

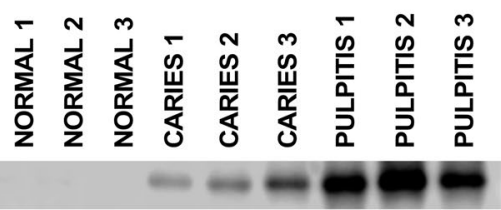

cGAS

STING

GAPDH

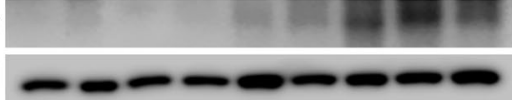

b

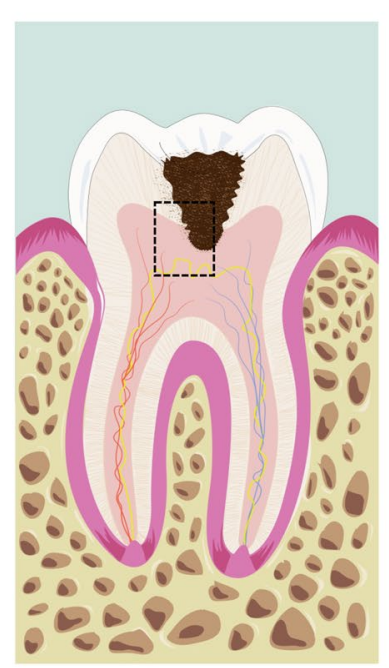

c

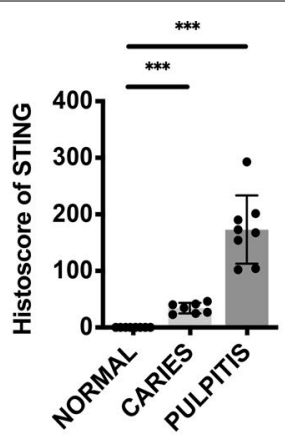

STING

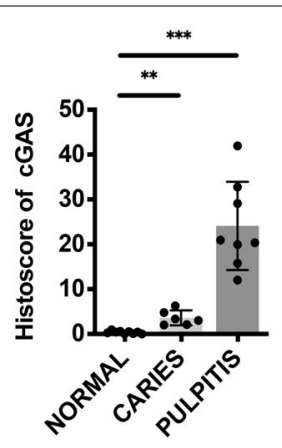

cGAS
20X

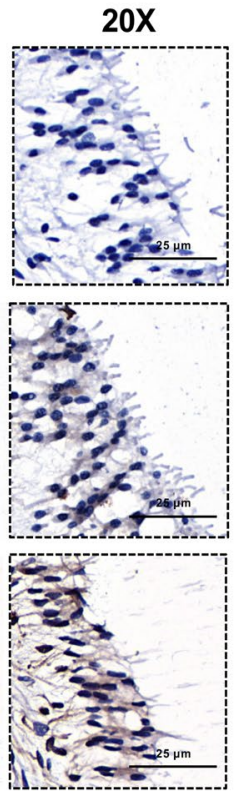

$5 X$

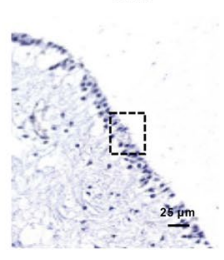

$20 X$
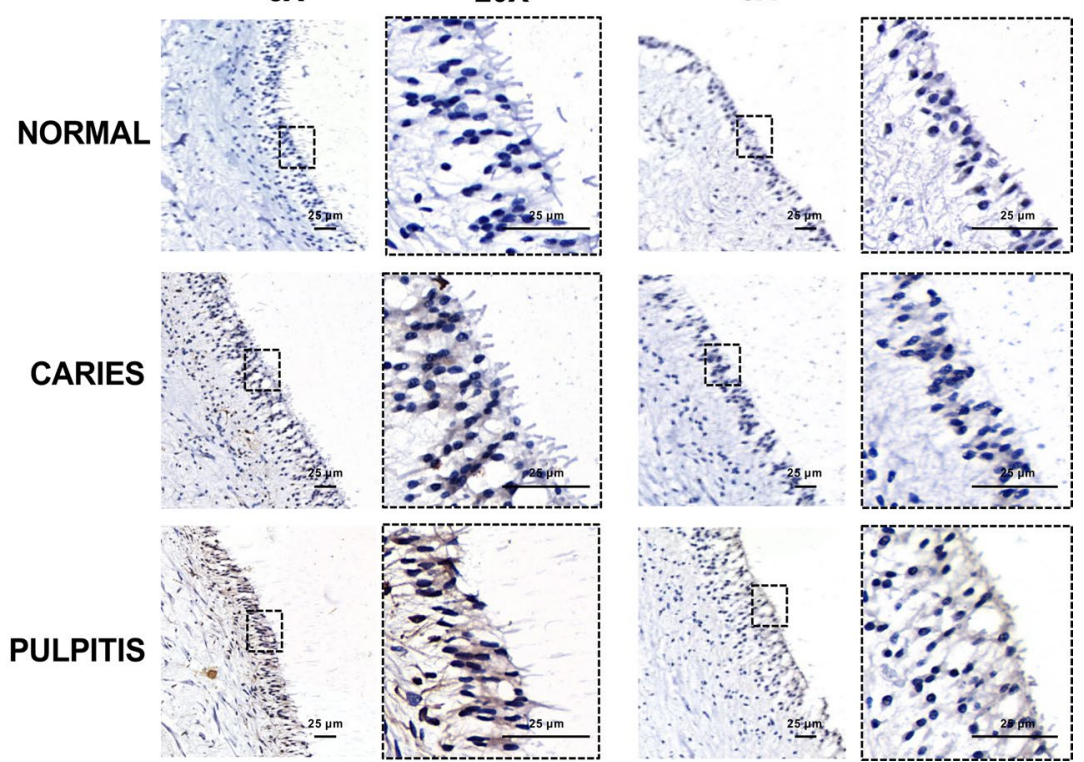

Fig. 1 High expression levels of CGAS and STING in inflamed dental pulp. a The expression of CGAS and STING in normal tissues, caries tissues and pulpitis tissues was measured by western blotting $(n=3)$. $\mathbf{b}$ CGAS and STING expression in the odontoblast layer was visualized as a schematic model (left) by immunohistochemical (IHC) staining (right). Scale bar: $25 \mu \mathrm{m}$. c Histoscores of cGAS and STING were determined from cGAS and STING expression levels in normal tissues $(n=8)$, caries tissues $(n=7)$ and pulpitis tissues $(n=8)$. The data are the mean \pm SEM $\left({ }^{*} p<0.05,{ }^{* *} p<0.01\right.$ ***, $p<0.001)$ 

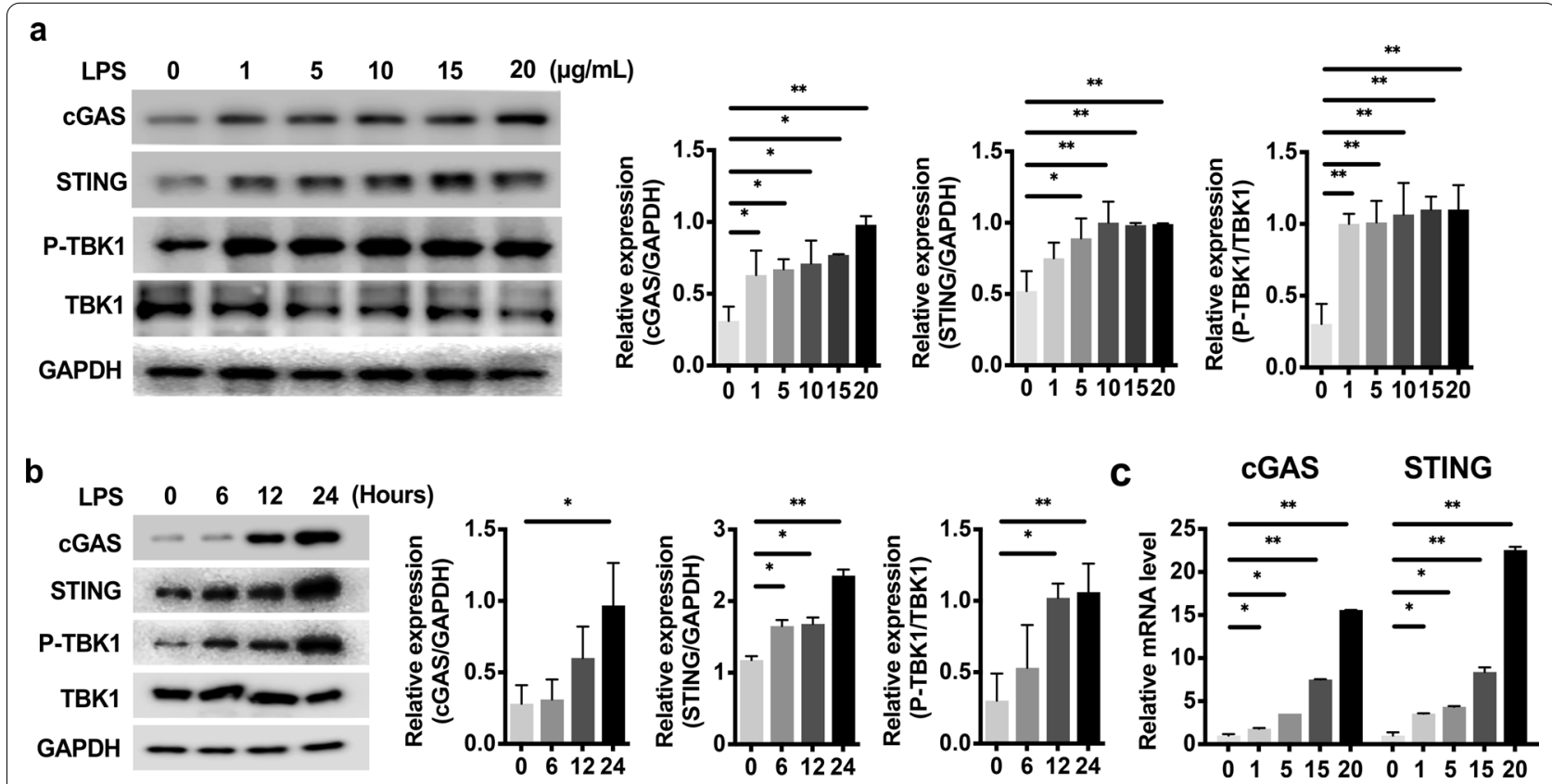

Fig. 2 The cGAS-STING pathway was activated by LPS in mDPC6T cells. $\mathbf{a}-\mathbf{b}$ The expression of cGAS and STING and the phosphorylation of TBK1 in $\mathrm{mDPC6T}$ cells were assessed by western blotting. mDPC6T cells were treated with different concentrations of LPS $(1,5,10,15$, and $20 \mu \mathrm{g} / \mathrm{ml})$ for $24 \mathrm{~h}(\mathbf{a})$ or $20 \mathrm{\mu g} / \mathrm{ml}$ LPS for $0,6,12$, and $24 \mathrm{~h}$ to induce inflammation (b). The quantitative data represent the relative ratio of the target protein to total TBK1 or GAPDH. c Real-time PCR was performed to quantify the relative mRNA levels of CGAS and STING in mDPC6T cells after $24 \mathrm{~h}$ of stimulation with different concentrations of LPS. The data are the mean \pm SEM, $n=3\left({ }^{*} p<0.05,{ }^{* *} p<0.01{ }^{* *} p<0.001\right)$

exacerbation of inflammation in patient tooth samples (Fig. 1b, c). These data demonstrated that the expression of cGAS and STING positively correlated with inflammation in caries and pulpitis tissues.

\section{The cGAS-STING pathway was activated by LPS in odontoblast-like cells}

To further understand the mechanism by which the cGAS-STING pathway promotes odontoblast inflammation, we evaluated changes in the expression of cGAS-STING pathway components in an LPS-stimulated odontoblast-like cell line (mDPC6T) in vitro. The expression of cGAS and STING was upregulated by LPS in mDPC6T cells in a dose-dependent (Fig. 2a) and time-dependent manner (Fig. 2b). mDPC6T cells were stimulated with $20 \mu \mathrm{g} / \mathrm{ml} \mathrm{LPS}$, and cGAS and STING expression was increased after $24 \mathrm{~h}$ compared with $6 \mathrm{~h}$ and $12 \mathrm{~h}$ (Fig. 2b). Real-time PCR confirmed that LPS activated cGAS and STING mRNA expression in mDPC6T cells (Fig. 2c). Consistent with this observation, the phosphorylation of the downstream molecule TBK1 also suggested that the cGAS-STING pathway was activated by LPS (Fig. 2a, b).

\section{cGAS-STING activation exacerbated LPS-induced mDPC6T} inflammation

To validate the effect of knocking down cGAS or STING on LPS-induced mDPC6T cells, we next analyzed the protein level of the downstream STING target protein TBK1 (Fig. 3a, b) and the mRNA expression level of proinflammatory cytokines (Fig. 3c, d). siRNAmediated knockdown of cGAS or STING significantly decreased the LPS-induced phosphorylation of TBK1 (Fig. 3a, b). In addition, the increased expression of IL-6 and CXCL10 induced by LPS was significantly reversed by cGAS or STING knockdown (Fig. 3c and d), suggesting that inhibiting cGAS or STING ameliorates odontoblast inflammation. However, IFN- $\alpha$ and $\beta$ were not induced by LPS treatment after cGAS or STING knockdown (Fig. 3c, d).

Furthermore, we also observed that cGAS or STING affected the nuclear translocation of p65 and IRF3. After knocking down STING in mDPC6T cells, we measured the expression of p65 and IRF3 in the nucleus and cytoplasm (Fig. 3e). The protein levels of p65 and IRF3 in the cytoplasm were increased after STING knockdown (Fig. 3e). Consistently, nuclear p65 and IRF3 protein levels were decreased (Fig. 3e). These results demonstrated that STING knockdown blocked the nuclear translocation of p65 and IRF3. 
(See figure on next page.)

Fig. 3 The CGAS-STING pathway regulated LPS-induced mDPC6T inflammation. a-b mDPC6T cells were transfected with anti-cGAS siRNA (a) or anti-STING siRNA (b) twice at an interval of $48 \mathrm{~h}$. LPS $(20 \mathrm{\mu g} / \mathrm{ml})$ was administered $24 \mathrm{~h}$ after the second transfection. The phosphorylation of TBK1 was analyzed by western blotting. The quantitative data represent the relative ratio of the target protein to total TBK1. c-d Real-time PCR was performed to detect the expression of IL-6, CXCL10, IFN- $a$ and IFN- $\beta$ in mDPC6T cells in response to 24 h of LPS stimulation without or with cGAS (c) or STING (d) knockdown. GAPDH served as the loading control. e Western blotting was performed to analyze the cytoplasmic and nuclear protein levels of p65 and IRF3 in STING-knockdown mDPC6T cells. $\beta$-tubulin served as the loading control for cytoplasmic proteins. Lamin A/C served as the loading control for nuclear proteins. The data are the mean $\pm S E M, n=3\left({ }^{*} p<0.05,{ }^{* *} p<0.01{ }^{* *} p<0.001\right)$

\section{LPS induced mitochondrial injury and mtDNA leakage into the cytosol in mDPC6T cells}

To elucidate the mechanism of the cGAS-STING pathway in LPS-induced odontoblast inflammation, we focused on the relationship between the cGASSTING pathway and mitochondrial dysfunction, which may cause mtDNA leakage into the cytosol. We found downregulated expression of the mitochondrial outer membrane proteins TOMM20 and VDAC1 after LPS challenge (Fig. 4a) [27, 28]. Moreover, the expression of the mtDNA-binding protein mitochondrial transcription factor A (TFAM) was also downregulated in inflamed mDPC6T cells (Fig. 4a) [13, 29]. To investigate mitochondrial function in inflamed mDPC6T cells, we evaluated mtROS by MitoSOX Red staining. Mitochondrial function was impaired in inflamed mDPC6 $\mathrm{T}$ cells, as indicated by the increased production of mtROS (Fig. 4b). In addition, in inflamed mDPC6T cells, intact mtDNA structures were observed by staining with an anti-dsDNA antibody (Fig. 4c). We observed that high levels of dsDNA translocated into the cytosol in inflamed mDPC6T cells and that some dsDNA colocalized with mitochondria (Fig. 4c). To confirm whether mtDNA was released into the cytosol, we quantified cytosolic mtDNA in mDPC6T cells with or without LPS stimulation. We found significant enrichment of cytosolic mtDNA in mDPC6T following LPS challenge (Fig. 4d).

\section{mtDNA leakage into the cytosol activated the CGAS-STING pathway in LPS-induced mDPC6T cells}

To determine the role of cytosolic mtDNA in the cGASSTING pathway, we used EtBr to block the replication of mtDNA but not nuclear DNA [30]. After the application of EtBr, mtDNA was depleted from mDPC6T cells, and the mtDNA copy number was reduced by approximately $80 \%$ (Fig. 5a). LPS-induced phosphorylation of TBK1 was restricted in mtDNA-depleted mDPC6T cells (Fig. 5b), and subsequently, the proinflammatory cytokines IL-6 and CXCL10 were markedly attenuated (Fig. 5c). To confirm the role of mtDNA in the nuclear translocation of p65 and IRF3, we analyzed the protein expression of p65 and IRF3 in the nucleus and cytoplasm in mDPC6T cells after treatment with EtBr. Cytosolic p65 and IRF3 levels were increased after $\mathrm{EtBr}$ treatment, as analyzed by western blotting (Fig. 5d). Consistently, nuclear p65 and IRF3 levels were decreased (Fig. 5d) compared with those in the control groups. These results showed that mtDNA depletion inhibited the nuclear import of p65 and IRF3 (Fig. 5d).

To confirm the regulatory effect of cytosolic mtDNA on STING in inflamed mDPC6T cells, we isolated mtDNA and transfected it into STING-knockdown mDPC6T cells. The transfected cytosolic mtDNA rescued the phosphorylation of TBK1, which was inhibited by STING knockdown (Fig. 5e), and induced the secretion of the proinflammatory cytokines IL-6 and CXCL10 (Fig. 5f). Release of mtDNA into the cytosol activated STING, resulting in elevated inflammation. In short, these results showed that leaked cytosolic mtDNA plays an important role in inflamed mDPC6T cells via the cGAS-STING pathway.

\section{Discussion}

This study uncovered a novel cellular response to mtDNA that engages the antimicrobial innate immune response in odontoblasts. Our results demonstrated that mitochondrial dysfunction led to mtDNA leakage, which activated the cGAS-STING pathway and the inflammatory cytokines IL-6 and CXCL10 in inflamed mDPC6T cells. Thus, therapeutic strategies targeting this pathway might be an effective way to protect the odontoblast barrier following cariogenic bacterial stimulation.

In our previous study, we found that autophagy was activated to regulate odontoblast differentiation [31] and that Parkin-dependent mitophagy was activated to degrade impaired mitochondria in LPS-induced odontoblasts [21]. Several studies have shown that the accumulation of abnormal mitochondria and cytoplasmic transport of mtDNA are activated by the consumption of autophagic proteins, which depend on the NALP3 inflammasome and mtROS [32]. In our study, we found that the expression of TOMM20 and VDAC1, which are located in the mitochondrial outer membrane and regulate mitochondrial permeability [27, 28], was significantly decreased after LPS stimulation (Fig. 4a). The expression of TFAM, which is the most abundant protein associated with mtDNA and is crucial for maintaining mtDNA 


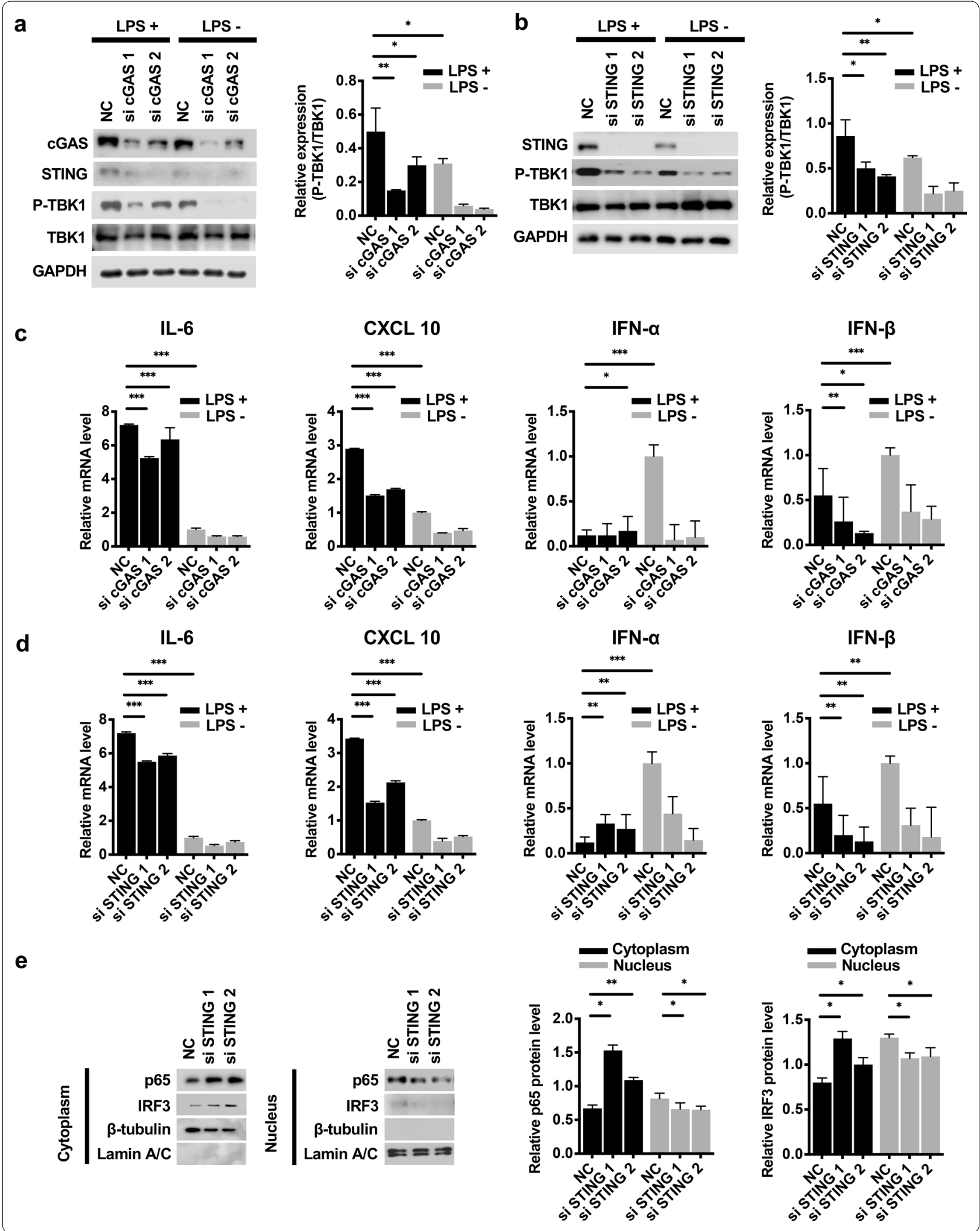




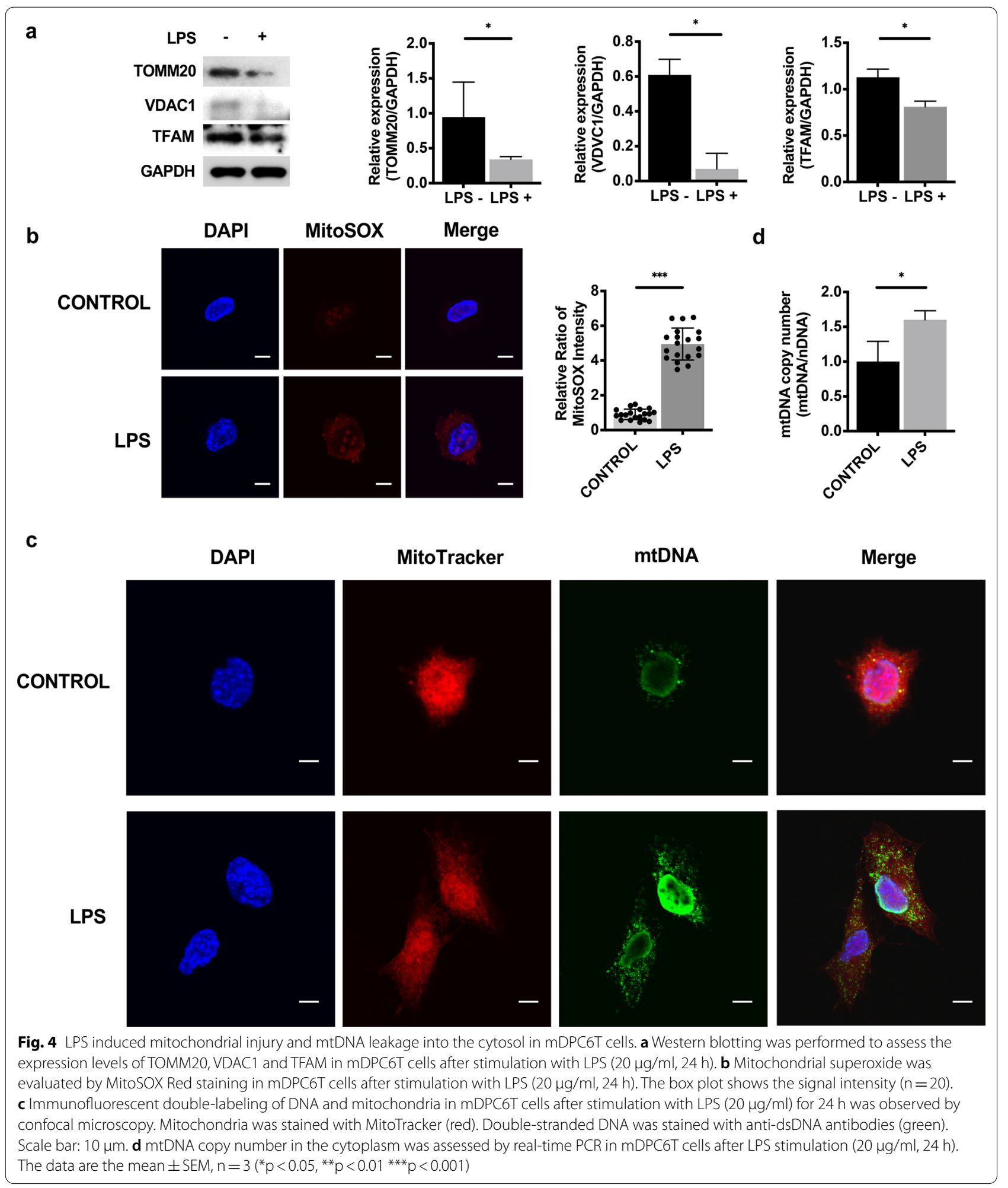

structure, transcription, and replication [13, 29], was significantly decreased by LPS stimulation (Fig. 4a). Moreover, we detected mtDNA leakage into the cytosol (Fig. 4c) after stimulation with LPS. To confirm the release of mtDNA, we quantified mtDNA in isolated cytosolic fractions by PCR (Fig. 4d). We found significant enrichment 


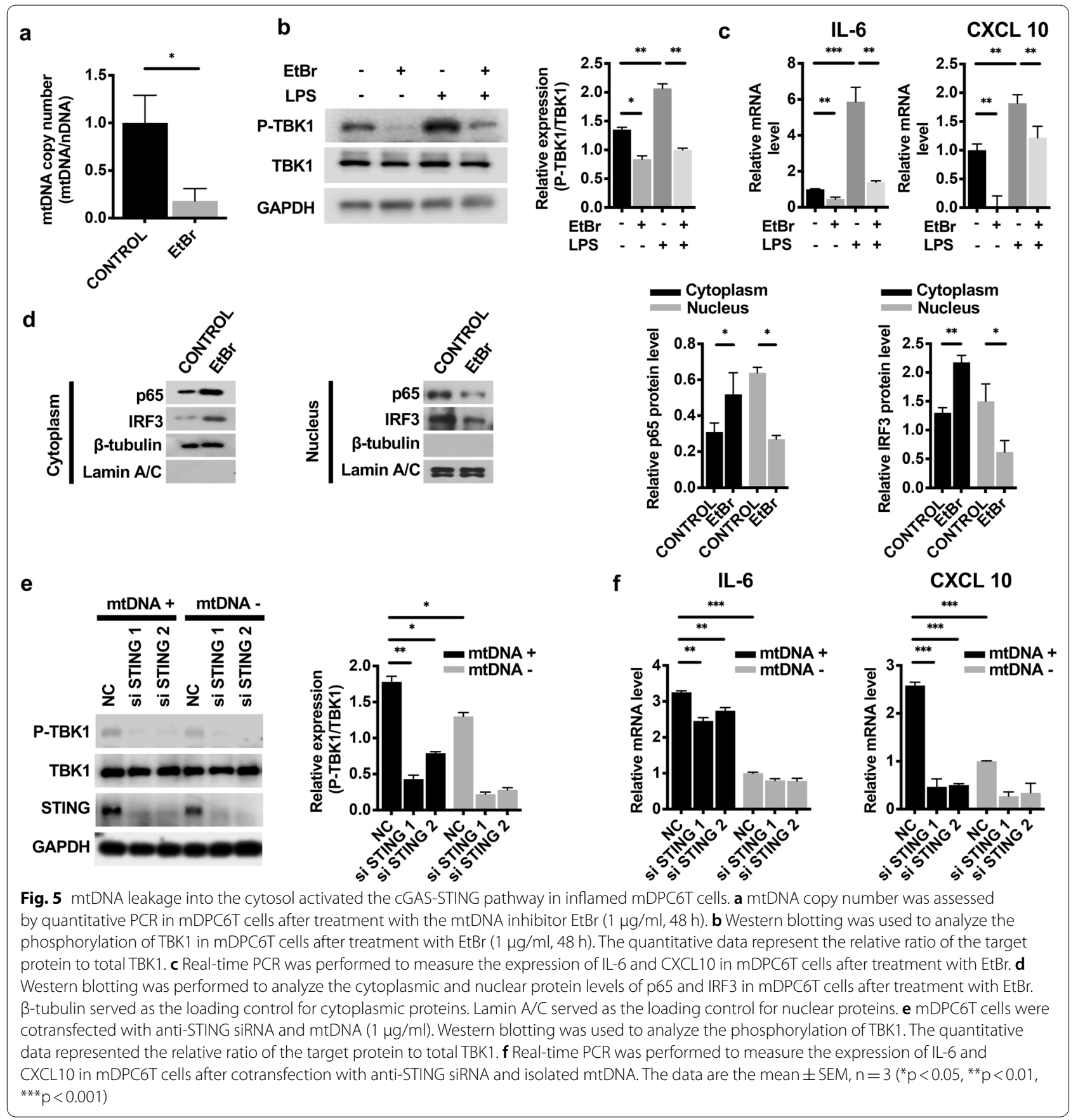

in mtDNA in the cytosol under LPS conditions compared with that in the control groups. These data demonstrated the possible relationship between mtDNA and the odontoblast inflammatory response. Mitochondrial damage was exacerbated by inflammation, which caused mtDNA release into the cytosol.

Various mitochondrial stresses including bacterial infection can lead to mtDNA release into cytosol. Infection with the bacterial pathogen such as mycobacterium tuberculosis can trigger cGAS and then stimulate IRF3dependent type I interferon response. Mitochondrial dynamics and cytosolic mtDNA contribute to IFN- $\beta$ induction by mycobacterium tuberculosis [33]. $\mathrm{H}_{2} \mathrm{O}_{2}$ induced by Streptococcus pneumoniae resulted in mitochondrial damage, mtDNA leakage and IFN type I production in alveolar epithelia cells [34]. Pathogen-infected cells also secrete IL- $1 \beta$ activating the cGAS-STING pathway in surrounding bystander cells. IL-1 $\beta$ stimulation 
of bystander cells promotes mitochondrial dysfunction, decreases mitochondrial membrane potential and induces mtDNA leakage into cytosol [35-37].

Emerging evidence suggests that mtDNA is a major inflammatory activator when it leaks from stressed mitochondria and can promote the progression of various inflammation-related diseases [11, 15, 16, 38]. In our study, we found that the depletion of mtDNA could restrict the secretion of the inflammatory cytokines IL-6 and CXCL10. These results provide evidence of a link between mtDNA and the odontoblast inflammatory response. This result suggested that mtDNA, as an important inflammatory driver, could participate in cellular mechanisms that contribute to the progression of odontoblast inflammation and affect the dentin-pulp complex that defends against microbial invasion.

Emerging evidence has shown that mtDNA acts as a cell-intrinsic cGAS ligand to trigger the STING signaling pathway in certain contexts to drive inflammation $[7$, $15,18]$. In our study, after utilizing the mtDNA inhibitor $\mathrm{EtBr}$, we found that phosphorylation of the STING pathway downstream mediators TBK1 was inhibited (Fig. 5b). mtDNA rescued the activation of TBK1 phosphorylation after STING knockdown (Fig. 5e). Depletion of mtDNA inhibited the nuclear translocation of p65 and IRF3 (Fig. 5d) and reduced the expression of IL-6 and CXCL10 (Fig. 5c). Therefore, our findings strongly suggested that mtDNA promoted odontoblast inflammation by activating cGAS-STING signaling and inducing inflammatory cytokine release. The mechanism by which $\mathrm{mtDNA}$ regulates the cGAS-STING pathway in inflamed odontoblasts in our study was consistent with that of a previous study in human tubular epithelial cells [39]. The role of the mtDNA-cGAS-STING axis in an animal model of pulpitis requires further investigation.

\section{Conclusions}

Our study revealed a previously unreported mechanism of the cellular immune response through the mtDNAcGAS-STING axis in odontoblast inflammation. These data suggest that therapeutic STING inhibitors may be used as anti-inflammatory drugs for pulpitis treatment.

\footnotetext{
Abbreviations

mtDNA: Mitochondrial DNA; cGAS: Cyclic GMP-AMP synthase; STING: Stimulator of interferon genes; EtBr: Ethidium bromide; TBK1:TANK-binding kinase
}

1; NF-kB: Nuclear factor kappa-B; IRF3: Interferon regulatory factor 3; nDNA: Nuclear DNA; PRRs: Pattern recognition receptors; mtROS: Mitochondrial reactive oxygen species; mtDAMPs: Mitochondrial danger-associated molecular patterns; TFAM: Mitochondrial transcription factor A; LPS: Lipopolysaccharide.

\begin{abstract}
Acknowledgements
Not applicable.

Authors' contributions

Lu Zhou: contributed to conception, design, data acquisition, analysis, and interpretation, drafted and critically revised the manuscript. Yi-Fei Zhang, Fu-Hua Yang and Han-Qing Mao: contributed to design, data acquisition and critically revised the manuscript. Zhi Chen: contributed to critically revised the manuscript. Lu Zhang: contributed to conception, design, data acquisition, analysis, and interpretation, drafted and critically revised the manuscript. All authors gave final approval and agree to be accountable for all aspects of the work.

\section{Funding}

This work was supported by National Natural Science Foundation of China $(81974148,81771064)$ to Lu Zhang.
\end{abstract}

Availability of data and materials

Not applicable.

\section{Declarations}

Ethics approval and consent to participate

This study was approved by the Institutional Ethics Board of Wuhan University.

Consent for publication

Not applicable.

\section{Competing interests}

The authors declared no conflict of interest.

\section{Author details}

${ }^{1}$ The State Key Laboratory Breeding Base of Basic Science of Stomatology (Hubei-MOST) and Key Laboratory of Oral Biomedicine Ministry of Education, School and Hospital of Stomatology, Wuhan University, Wuhan, China. ${ }^{2}$ Department of Endodontics, School and Hospital of Stomatology, Wuhan University, HongShan District, LuoYu Road No. 237, Wuhan 430079, China.

Received: 24 February 2021 Accepted: 13 April 2021

Published online: 20 May 2021

\section{References}

1. Disease GBD, Injury I, Prevalence C. Global, regional, and national incidence, prevalence, and years lived with disability for 328 diseases and injuries for 195 countries, 1990-2016: a systematic analysis for the Global Burden of Disease Study 2016. Lancet. 2017;390(10100):1211-59.

2. Kawashima N, Okiji T. Odontoblasts: Specialized hard-tissue-forming cells in the dentin-pulp complex. Congenit Anom (Kyoto). 2016;56(4):144-53.

3. Farges JC, Alliot-Licht B, Baudouin C, Msika P, Bleicher F, Carrouel F. Odontoblast control of dental pulp inflammation triggered by cariogenic bacteria. Front Physiol. 2013;4:326.

4. Ablasser, A. and Z.J. Chen, cGAS in action: Expanding roles in immunity and inflammation. Science, 2019. 363(6431).

5. Chen Q, Sun L, Chen ZJ. Regulation and function of the CGAS-STING pathway of cytosolic DNA sensing. Nat Immunol. 2016;17(10):1142-9. 
6. Motwani M, Pesiridis S, Fitzgerald KA. DNA sensing by the CGAS-STING pathway in health and disease. Nat Rev Genet. 2019;20(11):657-74.

7. Ishikawa H, Barber GN. STING is an endoplasmic reticulum adaptor that facilitates innate immune signalling. Nature. 2008;455(7213):674-8.

8. Hopfner KP, Hornung V. Molecular mechanisms and cellular functions of CGAS-STING signalling. Nat Rev Mol Cell Biol. 2020;21(9):501-21.

9. Tanaka, Y. and Z.J. Chen, STING specifies IRF3 phosphorylation by TBK1 in the cytosolic DNA signaling pathway. Sci Signal, 2012. 5(214): p. ra20.

10. Zhang X, Bai XC, Chen ZJ. Structures and Mechanisms in the CGAS-STING Innate Immunity Pathway. Immunity. 2020;53(1):43-53.

11. West AP, Shadel GS. Mitochondrial DNA in innate immune responses and inflammatory pathology. Nat Rev Immunol. 2017;17(6):363-75.

12. Shadel GS, Clayton DA. Mitochondrial DNA maintenance in vertebrates. Annu Rev Biochem. 1997:66:409-35.

13. Kang D, Kim SH, Hamasaki N. Mitochondrial transcription factor A (TFAM): roles in maintenance of mtDNA and cellular functions. Mitochondrion. 2007;7(1-2):39-44.

14. Shadel GS, Horvath TL. Mitochondrial ROS signaling in organismal homeostasis. Cell. 2015;163(3):560-9.

15. Riley, J.S. and S.W. Tait, Mitochondrial DNA in inflammation and immunity. EMBO Rep, 2020. 21(4): p. e49799.

16. Zhong F, Liang S, Zhong Z. Emerging Role of Mitochondrial DNA as a Major Driver of Inflammation and Disease Progression. Trends Immunol. 2019;40(12):1120-33.

17. Nakahira K, Hisata S, Choi AM. The Roles of Mitochondrial DamageAssociated Molecular Patterns in Diseases. Antioxid Redox Signal. 2015:23(17):1329-50.

18. Zhong Z, Liang S, Sanchez-Lopez E, He F, Shalapour S, Lin XJ, Wong J, Ding S, Seki E, Schnabl B, Hevener AL, Greenberg HB, Kisseleva T, Karin M. New mitochondrial DNA synthesis enables NLRP3 inflammasome activation. Nature. 2018:560(7717):198-203.

19. Lood C, Blanco LP, Purmalek MM, Carmona-Rivera C, De Ravin SS, Smith CK, Malech HL, Ledbetter JA, Elkon KB, Kaplan MJ. Neutrophil extracellular traps enriched in oxidized mitochondrial DNA are interferogenic and contribute to lupus-like disease. Nat Med. 2016;22(2):146-53.

20. Watson RO, Bell SL, MacDuff DA, Kimmey JM, Diner EJ, Olivas J, Vance RE, Stallings CL, Virgin HW, Cox JS. The Cytosolic Sensor CGAS Detects Mycobacterium tuberculosis DNA to Induce Type I Interferons and Activate Autophagy. Cell Host Microbe. 2015;17(6):811-9.

21. Yang F, Li Y, Duan H, Wang H, Pei F, Chen Z, Zhang L. Activation of mitophagy in inflamed odontoblasts. Oral Dis. 2019;25(6):1581-8.

22. Pezelj-Ribaric S, Anic I, Brekalo I, Miletic I, Hasan M, Simunovic-Soskic M. Detection of tumor necrosis factor alpha in normal and inflamed human dental pulps. Arch Med Res. 2002;33(5):482-4.

23. Lin H, Liu H, Sun Q, Yuan G, Zhang L, Chen Z. Establishment and characterization of a tamoxifen-mediated reversible immortalized mouse dental papilla cell line. Vitro Cell Dev Biol Anim. 2013;49(2):114-21.

24. Wang HS, Yang FH, Wang YJ, Pei F, Chen Z, Zhang L. Odontoblastic Exosomes Attenuate Apoptosis in Neighboring Cells. J Dent Res. 2019;98(11):1271-8

25. Li Y, Wang H, Pei F, Chen Z, Zhang L. FoxO3a Regulates Inflammationinduced Autophagy in Odontoblasts. J Endod. 2018;44(5):786-91.

26. Ishimoto, Y, R. Inagi, D. Yoshihara, M. Kugita, S. Nagao, A Shimizu, N Takeda, M. Wake, K. Honda, J. Zhou, and M. Nangaku, Mitochondrial
Abnormality Facilitates Cyst Formation in Autosomal Dominant Polycystic Kidney Disease. Mol Cell Biol, 2017. 37(24).

27. Chacinska A, Koehler CM, Milenkovic D, Lithgow T, Pfanner N. Importing mitochondrial proteins: machineries and mechanisms. Cell. 2009;138(4):628-44

28. Shimizu S, Narita M, Tsujimoto Y. Bcl-2 family proteins regulate the release of apoptogenic cytochrome $\mathrm{c}$ by the mitochondrial channel VDAC. Nature. 1999;399(6735):483-7.

29. West AP, Khoury-Hanold W, Staron M, Tal MC, Pineda CM, Lang SM, Bestwick M, Duguay BA, Raimundo N, MacDuff DA, Kaech SM, Smiley JR, Means RE, Iwasaki A, Shadel GS. Mitochondrial DNA stress primes the antiviral innate immune response. Nature. 2015;520(7548):553-7.

30. Hashiguchi K, Zhang-Akiyama QM. Establishment of human cell lines lacking mitochondrial DNA. Methods Mol Biol. 2009;554:383-91.

31. Pei, F., H.S. Wang, Z. Chen, and L. Zhang, Autophagy regulates odontoblast differentiation by suppressing NF-kappaB activation in an inflammatory environment. Cell Death Dis, 2016. 7: p. e2122.

32. Nakahira K, Haspel JA, Rathinam VA, Lee SJ, Dolinay T, Lam HC, Englert JA, Rabinovitch M, Cernadas M, Kim HP, Fitzgerald KA, Ryter SW, Choi AM. Autophagy proteins regulate innate immune responses by inhibiting the release of mitochondrial DNA mediated by the NALP3 inflammasome. Nat Immunol. 2011;12(3):222-30.

33. Wiens, K.E. and J.D. Ernst, The Mechanism for Type I Interferon Induction by Mycobacterium tuberculosis is Bacterial Strain-Dependent. PLoS Pathog, 2016. 12(8): p. e1005809.

34. Gao Y, Xu W, Dou X, Wang H, Zhang X, Yang S, Liao H, Hu X, Wang H. Mitochondrial DNA Leakage Caused by Streptococcus pneumoniae Hydrogen Peroxide Promotes Type I IFN Expression in Lung Cells. Front Microbiol. 2019;10:630.

35. Aarreberg, L.D., K. Esser-Nobis, C. Driscoll, A. Shuvarikov, J.A. Roby, and M. Gale, Jr., Interleukin-1beta Induces mtDNA Release to Activate Innate Immune Signaling via cGAS-STING. Mol Cell, 2019. 74(4): p. 801-815 e6.

36. Orzalli, M.H., A. Smith, K.A. Jurado, A. Iwasaki, J.A. Garlick, and J.C. Kagan, An Antiviral Branch of the IL-1 Signaling Pathway Restricts Immune-Evasive Virus Replication. Mol Cell, 2018. 71(5): p. 825-840 e6.

37. Mayer-Barber KD, Andrade BB, Oland SD, Amaral EP, Barber DL, Gonzales J, Derrick SC, Shi R, Kumar NP, Wei W, Yuan X, Zhang G, Cai Y, Babu S, Catalfamo M, Salazar AM, Via LE, Barry CE 3rd, Sher A. Host-directed therapy of tuberculosis based on interleukin-1 and type I interferon crosstalk. Nature. 2014:511(7507):99-103.

38. West AP. Mitochondrial dysfunction as a trigger of innate immune responses and inflammation. Toxicology. 2017:391:54-63.

39. Chung, K.W., P. Dhillon, S. Huang, X. Sheng, R. Shrestha, C. Qiu, B.A. Kaufman, J. Park, L. Pei, J. Baur, M. Palmer, and K. Susztak, Mitochondrial Damage and Activation of the STING Pathway Lead to Renal Inflammation and Fibrosis. Cell Metab, 2019. 30(4): p. 784-799 e5.

\section{Publisher's Note}

Springer Nature remains neutral with regard to jurisdictional claims in published maps and institutional affiliations.

Ready to submit your research? Choose BMC and benefit from

- fast, convenient online submission

- thorough peer review by experienced researchers in your field

- rapid publication on acceptance

- support for research data, including large and complex data types

- gold Open Access which fosters wider collaboration and increased citations

- maximum visibility for your research: over 100M website views per year

At $\mathrm{BMC}$, research is always in progress.

Learn more biomedcentral.com/submissions 